\title{
A survey on sleep assessment methods
}

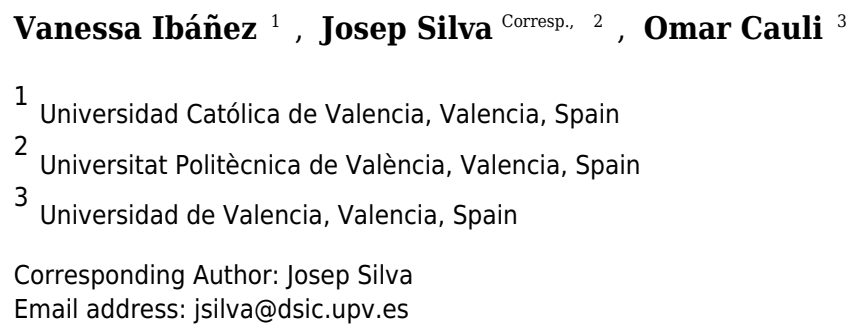

Purpose. A literature review is presented that aims to summarize and compare current methods to evaluate sleep.

Methods. Current sleep assessment methods have been classified according to different criteria; e.g., objective (polysomnography, actigraphy...) vs. subjective (sleep questionnaires, diaries...), contact vs. contactless devices, and need for medical assistance vs. self-assessment. A comparison of validation studies is carried out for each method, identifying their sensitivity and specificity reported in the literature. Finally, the state of the market has also been reviewed with respect to customers' opinions about current sleep apps.

Results. A taxonomy that classifies the sleep detection methods. A description of each method that includes the tendencies of their underlying technologies analyzed in accordance with the literature. A comparison in terms of precision of existing validation studies and reports.

Discussion. In order of accuracy, sleep detection methods may be arranged as follows:

Questionnaire < Sleep diary < Contactless devices < Contact devices < Polysomnography

A literature review suggests that current subjective methods present a sensitivity between $73 \%$ and $97.7 \%$, while their specificity ranges in the interval 50\%-96\%. Objective methods such as actigraphy present a sensibility higher than $90 \%$. However, their specificity is low compared to their sensitivity, being one of the limitations of such technology. Moreover, there are other factors, such as the patient's perception of her or his sleep, that can be provided only by subjective methods. Therefore, sleep detection methods should be combined to produce a synergy between objective and subjective methods. The review of the market indicates the most valued sleep apps, but it also identifies problems and gaps, e.g., many hardware devices have not been validated and (especially software apps) should be studied before their clinical use. 


\title{
A survey on sleep assessment methods
} Vanessa IBÁÑEZ ${ }^{\mathrm{a} *}$, Josep SILVA' ${ }^{\mathrm{b}}$, Omar CAULI $^{\mathrm{c}}$

${ }^{a}$ Nurse-Specialist in mental health. Associate professor in nursing and podology. Universidad Católica de Valencia, C.Espartero, 7, 46007 València, Spain

${ }^{b}$ Doctor in Computer Science. Associate professor in computer science Universitat Politècnica de València, Camino de Vera sn, 46022 València, Spain

${ }^{c}$ Doctor in nursing. Associate professor in nursing and podology. Research coordinator Universitat de València, Av. de Blasco Ibáñez, 13, 46010 València, Spain

All authors have seen and approved the manuscript.

All authors declare that no financial support exists for this work.

\begin{abstract}
All authors declare the absence of any conflict of interest defined as any financial interests or connections, direct or indirect, or other situations that might raise the question of bias in the work reported or the conclusions, implications, or opinions stated--including pertinent commercial or other sources of funding for the individual authors or for the associated departments or organizations, personal relationships, or direct academic competition for each author.
\end{abstract}


${ }^{a}$ Universidad Católica de Valencia, Valencia, Spain

${ }^{b}$ Universitat Politècnica de València, Valencia, Spain

${ }^{c}$ Universitat de València, València, Spain

\section{Abstract}

Purpose. A literature review is presented that aims to summarize and compare current methods to evaluate sleep.

Methods. Current sleep assessment methods have been classified according to different criteria; e.g., objective (polysomnography, actigraphy...) vs. subjective (sleep questionnaires, diaries...), contact vs. contactless devices, and need for medical assistance vs. self-assessment. A comparison of validation studies is carried out for each method, identifying their sensitivity and specificity reported in the literature. Finally, the state of the market has also been reviewed with respect to customers' opinions about current sleep apps.

Results. A taxonomy that classifies the sleep detection methods. A description of each method that includes the tendencies of their underlying technologies analyzed in accordance with the literature. A comparison in terms of precision of existing validation studies and reports.

Discussion. In order of accuracy, sleep detection methods may be arranged as follows:

$$
\text { Questionnaire }<\text { Sleep diary }<\text { Contactless devices }<\text { Contact devices }<\text { Polysomnography }
$$

A literature review suggests that current subjective methods present a sensitivity between $73 \%$ and $97.7 \%$, while their specificity ranges in the interval $50 \%-96 \%$. Objective methods such as actigraphy present a sensibility higher than $90 \%$. However, their specificity is low compared to their sensitivity, being one of the limitations of such technology. Moreover, there are other factors, such as the patient's perception of her or his sleep, that can be provided only by subjective methods. Therefore, sleep detection methods should be combined to produce a synergy between objective and subjective methods. The review of the market indicates the most valued sleep apps, but it also identifies problems and gaps, e.g., many hardware devices have not been validated and (especially software apps) should be studied before their clinical use.

\section{Introduction}

Sleep is fundamental to health. Sleep disorders can often be a symptom of a disease; or also may be an indicator of a future disease such as depression. For those reasons, sleep assessment is an essential component of any health check. As such, many health care systems stablish 
52

53

54

55

56

57

58

59

60

61

62

63

64

65

66

67

68

69

70

71

72

73

74

mechanisms to prevent sleep disorders by providing specific plans in relation to education and awareness of good sleep habits.

Over the years, many different sleep assessment methods have appeared. Specially in the last years, new methods have emerged with the appearance of new technologies such as mobile apps and novel advanced hardware sensors such as galvanic skin response measurers. In this survey, we review the current methods for the detection of sleep. From simple methods that only distinguish between awake or asleep states to complex methods able to distinguish all the sleep stages; from subjective methods such as sleep questionnaires and sleep diaries to objective methods such as polysomnography.

The main motivation of this survey is to produce a comprehensive and unbiased literature review from which we can extract a complete classification of sleep assessment methods (including new technologies such as mobile apps). There have in the past been different reviews of sleep assessment methods, but most of them are outdated (see, e.g., [1,2,3]), or they are partial, or only focus on a specific subset of methods (e.g., sleep questionnaires $[4,5,6,7,8,9]$, mobile apps [10,11], or contact sleep detection methods [12,13,14], etc.).

The survey was written to appeal to a range of people, who would have a broad spectrum of interests. It covers all sleep detection methods and, for each method, it also provides a table with the most used market products. Hence, because the survey tackles different technical areas, all technical terms have been conveniently introduced and explained. In all cases, explanations are supported and complemented with adequate references. Of course, not all sleep detection methods have the same precision; in fact, some of them are completely subjective. Therefore, the comparison of methods deserves a critical view on validation. Thus, we also report on the reliability and validity of the methods analyzing previous comparisons and validation studies.

\subsection{Survey methodology}

The literature review begins with a planning phase. This phase formulates research questions and defines inclusion and exclusion criteria. This phase is followed by search and screening of primary studies.

\section{A. Research questions}

We formulated two research questions to identify the current state of the art in sleep assessment methods:

- What methods for sleep assessment have been developed?

This research question aims to provide an overview of the sleep assessment methods, with special emphasis on those that have been developed over the last 10 years.

- What are the main characteristics of each sleep assessment method? This question complements the previous one, giving a deeper understanding of the sleep assessment methods.

B. Search process

The purpose of a literature review is to conduct a review of relevant studies to assess the body of knowledge that exists to support addressing the research questions. This process is rigorous and unbiased, and it involves a wide coverage of sources, such as online databases, journals, and 
92 conferences. The search string created to retrieve information from the electronic resources and 93 databases is the following:

94

95

96

97

98

99

100

101

102

103

104

105

106

107

108

109

110

111

112

113

114

115

116

117

118

119

120

121

122

123

124

125

126

127

(assessment OR evaluation OR detection)

AND (method OR tool OR environment OR system)

AND (sleep)

This search string was designed after an analysis of the keywords from the relevant literature, which was found from several general searches in the resources outlined above.

With the search terms defined, we started the process of identifying relevant literature in the following electronic databases: PubMed, LILACS, TOXNET, SCOPUS, ScienceDirect, and Google Scholar. Initially, we sought potential primary studies in the databases. In PubMed Health, the search string produced 1784 results. Therefore, we had to filter the results by refining the search string for that database:

\section{"sleep detection"[Title/Abstract] OR "sleep assessment"[Title/Abstract]}

As a result of the search process, 318 studies were identified. Excluding unavailable and duplicated results, we obtained 212 studies.

C. Inclusion and exclusion criteria

To address the research questions, the following inclusion and exclusion criteria were defined:

- IC1: Those papers that discussed sleep assessment methods were included.

- IC2: Those papers that described the characteristics of a sleep assessment method were included.

- EC1: Those papers that did not describe a sleep assessment method were excluded.

D. Studies Selection

Initially, we performed screening on the titles and abstracts to decide whether to include or exclude each study. As a result, from the 6 sources that we searched, a total of 114 studies were selected and 98 were excluded. We read in detail the full text of each primary study included in the preliminary selection to decide whether to include or exclude the study. The primary studies included in the final selection correspond to the relevant papers that meet the research questions set out in this study. The QUOROM flow chart of the reviewing process is depicted in Fig. 1.

\section{E. Data extraction}

With the final set of primary studies decided upon, the data extraction activity was carried out on included papers. For each paper, we identified the kind of article (review, opinion, study, tool description...) and the sleep assessment methods it described. We grouped the data by sleep assessment methods and identified a total of 5 categories where all sleep assessment methods can be classified (see Section 2). For each method, a single document was produced, grouping the data coming from all papers related to the tool. This produced summaries and charts that helped us to study and classify the methods. 


\subsection{Structure of the survey}

129 The rest of the paper has been structured as follows: in Section 2, a classification of sleep

130 detection methods is proposed. Then, in Section 3, those methods that need medical assistance

131 are explained. Similarly, those methods that do not necessarily need medical assistance (self-

132 assessment methods) are explained in Section 4. In Section 5, we discuss the accuracy and

133 validity of the methods presented. We also discuss the usefulness of some of the methods, and we

134 comment on future developments. Finally, in Section 6 we provide a concluding summary.

\section{2. Classification of sleep detection methods}

136 Essentially, a sleep detection method is a function that classifies the sleep state of a patient. Most

137 sleep detection methods such as wrist actigraphy or mobile apps consider a binary function,

138 where the state can be classified as Awake / Sleep. More sophisticated methods consider a ternary

139 function: Awake / NREM / REM. And, finally, the most advanced methods, such as

140 polysomnography — often used as the gold standard-, consider a quinquenary function: Awake /

$141 \mathrm{N1} / \mathrm{N2} / \mathrm{N} 3$ / REM. Hence, any method can produce a two-dimensional chart where the $\mathrm{X}$-axis

142 is Time, and the Y-axis is the State of the Patient. In the particular case of polysomnography, the

143 Y-axis has five possible values; thus, it can determine the sleep stage of the patient at any time,

144 and study the transitions occurring between the states. Of course, a sleep study such as a

145 polysomnography often produces much more complementary information that can be used, e.g.,

146 to diagnose sleep diseases. Among the information reported by a polysomnography we find

147 oxygen saturations, limb movements, apneas, respiratory events by body position, etc. The

148 interested reader is referred to $[15,16,17]$ for information about sleep study reports and their

149 interpretation and usage.

150 The information that is common to the majority of sleep detection methods is the one that

151 refers to a binary state classification (i.e., Awake / Sleep), because this is achieved by the basic

152 methods, and subsumed by the advanced methods. Table 1 defines the basic parameters that can

153 be collected by a binary state classification method. In grey, we show the primary data that

154 should be collected by the sleep detection device and, in white, we show the most important

155 parameters that can be derived from the primary data.

156 These parameters are particularly useful to determine the kind of sleep of patients, and each

157 single parameter is relevant for a different sleep disorder or disease. For instance, the sleep onset,

158 sleep latency, and total sleep time are essential to diagnose patients with insomnia. Similarly, an

159 excess in the awakening and arousal indices suggests increased sleep fragmentation. In addition

160 to the number of sleep states that they are able to detect, a sleep detection method can be

161 classified according to other functional and operational characteristics, such as their underlying

162 technology, which in turn directly affects their precision and validity.

163 In Fig. 2, we present a taxonomy of sleep detection methods. They all can be classified into 164 two main groups according to whether they need medical assistance (Medical Assistance) or not 165 (Self-Assessment). In this respect, there are methods that have been classified as not requiring 166 medical assistance, such as Questionnaires and Sleep Diaries, even though their interpretation 167 should be normally done by a professional. However, in the current state of the art there are 168 many systems such as mobile apps that provide custom sleep questionnaires and produce reports 169 without medical assistance. Hence, they are classified as Self-Assessment. They both deserve a 170 deep discussion and will be explained separately in Sections 3 and 4, respectively. 
Self-Assessment methods include subjective methods such as questionnaires and sleep diaries (the figure lists some instances), and objective methods based on hardware sensors, which in turn can be classified as Contact devices or Contactless devices, depending on whether they need to be in contact with the patient's body during sleep. Those devices that are based on the echo produced by signals can be further classified into Sonar, Radar, and Lidar devices. All of them will be explained in a dedicated section.

\section{Medical Assistance Methods}

There are different studies that can be performed in a sleep laboratory. All of them have one significant advantage and one significant disadvantage that differentiate them from the home detection methods. The obvious advantage is that these methods can use advanced technology such as electroencephalograms, electrocardiograms, etc. that cannot be used at home. The advantage of these methods is that they can be extremely precise, and can be discrete (e.g., are able to distinguish between sleep phases). For this reason, these methods have been often used as the gold standard for sleep evaluation (see, e.g., $[4,5,6,18,9,19])$. Of course, the use of this exclusive technology comes with a cost: these methods are expensive, time-consuming, require professional assistance and, often, they can only be done for a reduced period of time (e.g., one or two days). But, additionally, there is another important functional disadvantage: the assessment made by these methods is done in a context that is not the usual sleep context of the patient (i.e., a sleep clinic or a hospital) and, thus, a normal sleep situation is not measured.

\subsection{Polysomnogram (PSG)}

The term polysomnogram comes from the Greek root poly (many), the Latin noun somnus (sleep), and the Greek verb noun gramma (drawing or diagram). A PSG [15,16,17] is a medical procedure composed of several concurrent but independent tests that monitor different body functions during sleep and that are recorded for their later study using different channels. An exhaustive list of tests and information gathered in a modern PSG follows:

- Electroencephalogram (EEG) - measures and records the brainwave activity to identify sleep stages and detect seizure activity.

- Electrooculogram (EOG) - records eye movements. These movements are important for identifying the different sleep stages, especially the REM stage.

- Electromyogram (EMG) - records muscle activity (e.g., teeth grinding and face twitches; but also, limb movements using surface EMG monitoring of limb muscles, periodic or other). Chin EMG is necessary to differentiate REM from wakefulness, limb EMG can identify periodic limb movements during sleep (PLMS).

- Electrocardiogram $(E K G)$ - records the heart rate and rhythm.

- Pulse oximetry - monitors the oxygen saturation $\left(\mathrm{SO}_{2}\right)$.

- Respiratory monitor - measures the respiratory effort (thoracic and abdominal). It can be of several types, including impedance, inductance, strain gauges, etc.

- Capnography - measures and graphically displays the inhaled and exhaled $\mathrm{CO}_{2}$ concentrations at the airway opening.

- Transcutaneous monitors - measure the diffusion of $\mathrm{O}_{2}$ and $\mathrm{CO}_{2}$ through the skin.

- Microphone - continuously records the snoring volume and kind.

- Video camera - continuously records video. It is useful to identify the body motion and position. 
214

215

216

217

218

219

220

221

222

223

224

225

226

227

228

229

230

231

232

233

234

235

236

237

238

239

240

241

242

243

244

245

246

247

248

249

250

251

252

253

254

255

256

- Thermometer - records the core body temperature and its changes.

- Light intensity tolerance test - determines the influence of light intensity on sleep.

- Nocturnal penile tumescence test - is used to identify physiological erectile dysfunctions.

- Esophageal tests - includes pressure manometry, to measure pleural pressure; esophageal manometry to assess peristalsis, and esophageal $\mathrm{pH}$ monitoring (acidity test).

- Nasal and oral airflow sensor - records the airflow and the breathing rate.

- Gastroesophageal monitor - is used to detect Gastroesophageal Reflux Disease (GERD).

- Blood pressure monitor - measures the blood pressure and its changes.

Depending on the particular sleep study that needs to be performed, only some specific tests from the above list are generally selected - and they are also parameterized for each specific case. For instance, the EEG is usually comprised of 10-16 electrodes, but in patients with epilepsy, often 20 electrodes are used. Similarly, to assess bruxism, the EMG electrodes can be placed over the masseter muscle, but to assess other sleep disorders, the EMG electrodes are placed in other muscle groups. For example, the intercostal EMG is used to measure the effort during respiration.

Nowadays, the PSG is the most advanced tool for the diagnosis of many sleep disorders. According to [16] and [17], the main disorders that a PSG can evaluate are those in Fig. 3 (they are classified following the International Classification of Sleep Disorders [20]), being especially frequent (i) sleep apnea or another sleep-related breathing disorder, (ii) periodic limb movement disorder, (iii) narcolepsy, (iv) REM sleep behavior disorder, (v) unusual behaviors during sleep, and (vi) unexplained chronic insomnia.

\subsection{Multiple sleep latency test (MSLT)}

This sleep study [21,22] is a test to identify excessive daytime sleepiness (i.e., feeling sleepy in a situation where one should be awake and alert, e.g., driving a truck) and determines how long it takes the patient to fall asleep. It also identifies the phases of the sleep (e.g., how quickly and how often the patient enters REM sleep). MSLT is the standard test to diagnose idiopathic hypersomnia and narcolepsy, and it measures how quickly a patient falls asleep during the day in a quiet environment. An MSLT often starts the morning following a PSG and it lasts one complete day. The patient tries to sleep in five scheduled naps separated by two-hour breaks. For this reason, this test is often called a "nap study".

Each nap trial takes place in a quiet bedroom. The patient is connected with sensors to a device that can detect sleep stages. The standard procedure often includes an EEG, EOG, EMG, and EKG [21]. The equipment is composed of different electrodes and monitors:

$\circ$ Wires with small cup electrodes attached to the scalp with a conductive paste to measure brain activity (EEG). This detects in what stage of sleep is the patient.

$\circ$ Wire electrodes that are taped to the face near the eyes (EOG) and chin to show muscle activity (EMG).

- 2 elastic belts around the chest and stomach to measure breathing effort.

- A nasal cannula and small heat monitor to measure all breathing activity.

- A wire electrode on each leg to measure body movement/muscle activity.

○ A monitor taped to a finger to detect oxygen levels.

o 2-3 lead EKG monitors to show heart rate and rhythm.

○ A small microphone applied to the throat to detect snoring. 
Hence, the MSLT can identify exactly when the patient falls asleep, and whether or not they entered REM sleep. If the patient falls asleep, they are awakened after 15 minutes. The nap trial also ends if the patient does not fall asleep within 20 minutes. Patients with narcolepsy often have two or more REM periods during the MSLT. People with idiopathic hypersomnia fall asleep easily but do not reach REM sleep during the nap trial.

\subsection{Maintenance of wakefulness test (MWT)}

This test $[23,24]$ is performed over a whole day. Contrary to a PSG, this test is made while the patient is awake. Essentially, it challenges patients to attempt to stay awake during periodic tests. Therefore, an MWT may be helpful in the management of sleepy patients, particularly for driving purposes. It measures how alert a patient is during the day and it determines whether a patient is able to stay awake for a period of time in a quiet and relaxing environment. During the test, there are 4-5 periods of around 40 minutes each, spaced apart by 2 hours, where the patient is asked to stay relaxed in a quiet, faintly-lit bedroom. The first trial often begins 1.5 to 3 hours after the patient's normal wake-up time. The patient eats breakfast one hour prior to the first relaxing period and they have lunch after the second period. Between the periods, the patient can read the newspaper, watch TV, have a meal, or move freely inside the building, but they cannot go outside because daylight is a factor that must be eliminated during the test.

During the relaxing periods, patients are connected to a set of leads that monitor (i) heart activity with 2-3 ECG leads, (ii) brain activity with 4 EEG leads, (iii) chin muscle activity with 3 leads, and (iv) left and right eye movements. If the patient falls asleep for 90 seconds at any time during the relaxing period, the test is terminated. All data collected are analyzed by a sleep specialist to determine the patient's level of sleepiness during the day.

\subsection{CPAP titration test (CTT)}

A CTT [25] is a type of sleep study that is used to calibrate continuous positive airway pressure (CPAP) and bi-level positive airway pressure (BIPAP) therapies. CPAP/BIPAP are the common treatments in some sleep-related respiratory disorders (see Fig. 3)

such as central sleep apnea (BIPAP) and obstructive sleep apnea (CPAP), which eliminate breathing pauses during sleep. Before starting these treatments, a CTT is needed.

The objective of a CTT is to determine the amount of air pressure needed to prevent the upper airway from becoming blocked. This is studied during the sleep of the patient with a nasal mask that periodically changes the air pressure, and different sensors that monitor the sleep in a similar way to a PSG (i.e., they record oxygen levels, breathing, heart rate, brain waves, and leg and arm movements).

\subsection{Home Sleep Test (HST)}

The HST [26,27] is a kind of limited PSG that is made at home (i.e., portable equipment is transported to the patient's home). The number of channels used is often reduced to three: airflow, respiratory effort, and oximetry. It provides an indication only for high suspicion of obstructive sleep apnea - not other sleep disorders - and it has the obvious advantage that the context in which the sleep is evaluated is the normal one. The main disadvantages are that it cannot determine sleep stage, hypopneas, or arousals; and no one is present to replace leads. 


\section{Self-Assessment Methods}

298

299

300

301

302

303

304

305

306

307

308

309

310

311

312

313

314

315

316

317

318

319

320

321

322

323

324

325

326

327

328

329

330

331

332

333

334

335

336

337

338

\subsection{Sleep Questionnaires}

The preliminary evaluation of sleep in primary care is often completed with a sleep questionnaire (also known as a sleep scale). Sleep questionnaires are a very inexpensive and rapid test, and for these reasons, they are ideal for the first diagnostic test. Moreover, they summarize in a quantitative way the (subjective) perception of the patient about his or her own quality of sleep. Precisely because they are mostly subjective, sleep questionnaires can be influenced by the same sources of bias and inaccuracy as any other such reports. However, their subjectivity does not necessarily render questionnaires inaccurate, as it has been demonstrated by several validation studies (see $[4,6,5,18,7,9])$.

In general, filling in a sleep questionnaire does not require the assistance of sanitary professionals. They can be self-administered at any moment, even at home. For instance, the Google play's Sleep Apnea Screener is a mobile app that automatically provides a report after completing a questionnaire. Therefore, sleep questionnaires can be used by people (e.g., with sleep apnea) as a sleep control that can alert them about the need for a proper diagnosis provided by specialists.

Table 2 shows (in chronological order of appearance) the most extended sleep questionnaires used along the last 30 years. For a long time, we have been collecting all of them (some of them are not available online), and we have created a public repository where they all can be downloaded:

\section{http://users.dsic.upv.es/ jsilva/Sleep/}

For each questionnaire, the table shows:

- Its structure: number of questions/items and the scale used for the answers.

- The period of time that the questionnaire evaluates: if it is unspecific or unspecified it uses "Recently", if one or more questions refer to future or hypothetical situations it uses "Future" (e.g., "If I use CPAP I will feel better", "I would use CPAP, even if I had to pay for some of the cost", etc.).

- The percentage of objective questions in the questionnaire: a question that is (partially) subjective or that depends on memory is considered subjective. Only questions that are totally objective are considered objective (e.g., "have you taken drugs to sleep?", "how much do you weight?", etc.). The level of objectivity is indicated with a 1-4 scale, where 0 means close to $0 \%, 1$ means close to $25 \%, 2$ means close to $50 \%, 3$ means close to $75 \%$, and 5 means close to $100 \%$.

It is important to note that, although the goal of some questionnaires (e.g., PSQI) is to evaluate sleep quality (such as PSG, and actigraphy), others assess concepts distinct from sleep quality. For example, FOSQ measures the concept of sleepiness, which may or may not be related to sleep quality. Treating objective and subjective measures related to some aspect of sleep as evaluating sleep quality would ignore the fundamental concept on which they were developed and the principle that you select a measure based on the concept you are measuring for alignment and accuracy. Therefore, some questionnaires are fundamentally incomparable, and the selection of one questionnaire should be based on the purpose of each specific questionnaire. Table 3 summarizes the objective of each questionnaire. 
Having such an availability of different questionnaires (as shown in Table 2, their number of questions and scales vary a lot), the natural question is: "Which sleep questionnaire should I use?" Of course, those questionnaires with less questions are easier to administer, but those questionnaires with more questions collect more information. However, the question remains for those questionnaires with the same number of questions (e.g., SACS, SQ, SFV, OSA50). This question has motivated several studies to compare their sensitivity (true positive rate) and specificity (true negative rate). Some important studies comparing sleep questionnaires for the identification of sleep apnea are summarized in Table 4. The interested reader is referred to [28] for a survey on sleep questionnaires.

\subsection{Sleep Diaries}

Sleep diaries allow patients to self-assess their sleep. Sleep diaries have one important advantage over sleep questionnaires. While sleep questionnaires are filled in once, sleep diaries are filled in over a period of time (usually one or two weeks). This means that sleep diaries contain more information, and also that the information contained is more precise. This happens because a sleep questionnaire provides an overall perception, often ignoring the details, and it is highly dependent on the patient's memory because they summarize information about the previous one or two weeks. Contrarily, the sleep diary collects data every day, so that good and bad days are recorded. Moreover, the sleep diary is not so dependent on memory, because they are often filled in just after waking up. We have been collecting sleep diaries from hospitals, sleep centres and different studies. In our repository, there are more than 25 sleep diaries. The most representative are shown in Table 5. We have made them publicly available at:

\section{http://users.dsic.upv.es/ jsilva/Sleep/}

The Pittsburgh Sleep Diary [29] is the oldest sleep diary in our records (although there is evidence that sleep diaries were in clinical use for decades before its 1994 publication [30]). After it was proposed, many other diaries have been defined by researchers, hospitals, and sleep centres. In March 2005, 25 researchers attending the Pittsburgh Assessment Conference developed an initiative to compare a collection of sleep diaries in order to extract the best from each diary studied and integrate all together, producing an improved sleep diary. As a result, they proposed the "Consensus Sleep Diary" [31] (see Table 5). The diaries included in Table 5 are classified according to the information required from the user. In particular, they include different questions about their sleep such as time used to fall asleep, the amount and kind of food in the dinner, or use of drugs, etc. Specific information about the structure and information gathered by sleep diaries can be found at [28].

There is also the existence of sleep diaries that are distributed as mobile apps. The most used and better valued sleep diaries according to Google Play are Sleep Diary Pro (423 reviews with a mark of 4.2/5), Healthy Sleep Diary (223 reviews with a mark of 3.9/5), and Sleep Diary Lite (2263 reviews with a mark of 3.8/5). Tonetti L. et al. [32] compared the use of paper and electronic sleep diaries and concluded that they are similar with respect to their diagnostic power. 


\subsubsection{Contactless hardware devices to detect the sleep}

380

381

382

383

384

385

386

387

388

389

390

391

392

393

394

395

396

397

398

399

400

401

402

403

404

405

406

407

408

409

410

411

412

413

414

415

416

417

418
Contactless methods to assess sleep use one or more of the following technologies: Microphone (in [33] there is an informative discussion about how to quantify snoring and sleep apnea severity), video camera, (infrared) thermometer, pressure strap or belt, pillow or mattress accelerometer, echo-based devices [34] such as sonar, radar, or also lidar (still under development).

There exist periodic expert's reviews (see, e.g., $[35,14,36]$ ) that rank the most valued contactless devices according to the market (e.g., Amazon reviews). However, currently the most used device to assess the sleep is the smartphone. Because a smartphone contains a microphone, a camera, and an accelerometer, it can use these hardware features to monitor sleep. This has promoted the appearance of many mobile apps to assess sleep. The main contactless sleep detection apps according to the number of reviews in Google Play are shown in Table 6. Even though several experts' reviews (see, e.g., [37,13]) report high performance and reliability of these apps, there are still few scientific validation studies that support this claim. In contrast, several studies report that mobile apps are not yet prepared for clinical use [12,11,38,39]. For instance, a recent study [38] performed with 25 children (ages: 2-14) and where a smartphone recorded data simultaneously with a PSG suggested that smartphone apps may have value in increasing the user's awareness of sleep issues but would not yet be accurate enough to be used as a clinical tool.

\subsubsection{Contact hardware devices to detect the sleep}

Contact hardware devices to assess sleep are small devices that can be attached to the wrist, chest, ankle, or head. Some of these devices use the Cartesian representation to record the activity of the body and thus they are known as actigraphs. Most actigraphs use an accelerometer to register movements. The information collected is used to analyse sleep. Even though contemporary actigraph devices are electronic, the first actigraphs were mechanical (contrary to common belief). In fact, the first actigraphs date from the 1950s [40].

Due to the usefulness of the information collected by actigraphs, the use of actigraphy has been included in the ICSD-3 diagnostic criteria for several circadian sleep-wake rhythm disorders. Even though there is a clear continual improvement in the precision of sensors, and in the accuracy of algorithms, the use of actigraphs for clinical diagnosis should be considered when the device and algorithm used have been validated. In particular, the algorithm used to interpret the data is of major importance, because many proprietary algorithms do not pass enough quality controls, and some of them are even worse than the human inspection of the actigraphy data (see, e.g., [41]).

The performance and reliability of hardware devices have been compared by experts' reviews (see, e.g., [13]) and also by validation studies [42,12]. However, it is important to note that fitness trackers and phone apps tend to underestimate sleep disruptions and overestimate total sleep times and sleep efficiency in normal subjects [12]. 


\section{A critical discussion about accuracy and validation}

420 Each sleep detection method has its own level of reliability and precision. If we place the presented methods in order of accuracy, as reported in the literature $[41,42,28]$, we have:

$$
\text { Questionnaire }<\text { Sleep diary }<\text { Contactless devices }<\text { Contact devices }<\text { PSG }
$$

423

424

425

426

427

428

429

430

431

432

433

434

435

436

437

438

439

440

441

442

443

444

445

446

447

448

449

450

451

452

453

454

455

456

457

458

459

460

461

462

463
It is important to note that this formulation does not pretend to sort the methods according to their usefulness. It would be erroneous to state that self-reporting is inferior (or less useful) to more objective measures. This would fail to appreciate that data based on patient perception may be valuable in understanding sleep problems.

Being both mostly subjective, there is an important difference in the way that sleep diaries and questionnaires are completed. Questionnaires are filled in once, usually before the interview with the sleep therapist, thus, not just after waking up. In consequence, (1) the patient's memory strongly influences the quality of the information provided (he or she has to remember his or her sleep for a week or a month); and (ii) the information provided is a summary of many sleeps, thus, losing details about special days. In contrast, sleep diaries (1) are filled in every day, and (2) they are completed just after waking up. Hence, they are potentially more accurate and less influenced by the memory of the patient. Therefore, the amount of information and accuracy of sleep diaries is objectively superior to that of questionnaires. Here again, the superiority in precision of sleep diaries does not substitute the global assessment of sleep questionnaires, and the latter may be the relevant data therefore making the questionnaire more pertinent.

The accuracy of sleep questionnaires has been widely studied (see, e.g., [4,5,6,7,18,9,19], and see Table 4 where studies that evaluated sleep questionnaires are compared). All these studies used the PSG as a gold standard and tried to evaluate the sensitivity/specificity of the questionnaires in identifying sleep apnea. The sensitivity reported was in the interval $73.0 \%$ $97.6 \%$, while the specificity reported was in the interval $50 \%-96 \%$ (see Table 4 ). Most of the studies reported the STOP-BANG questionnaire as the one producing the best sensitivity. These studies are definitely useful, and provide good indicators, but our selection of a specific questionnaire must consider the specific illness and population targeted. Precisely because they target different populations the results of these studies are not always directly comparable. For instance, the study in [4] was performed on highway bus drivers in Turkey and the study in [9] only considered men, etc.

The effectiveness of sleep diaries has been evaluated in [43] and [32]. These studies also compare paper diaries and electronic diaries using an actigraph as the gold standard. Both studies found that, statistically, paper and electronic diaries collect the same data; thus, their accuracy and reliability is similar.

The main difference between contact and contactless devices is their underlying technology. In general, contact devices are more accurate because most of the sensors used to monitor sleep are strongly dependent on their distance from the patient (the closer the better). A good example are the accelerometers, which are sensors used in both contact devices such as wrist watches, and contactless devices such as mattress or pillow clips. It is fairly evident that it is much more reliable to directly measure the movements of the body than approximating them by measuring the movements of the mattress or pillow. The same happens with sonars, for example. A phone's microphone and speaker using ultrasounds as a sonar have an effective range of about 1 metre and its reliable distance is 0.5 metres. Of course, the results are more precise as the patient is closer. Unfortunately, the movements of the sonar can negatively affect its measurements and results, hence it is preferable putting it on a bedside table, lying still, instead of putting it on the 
464 mattress. As a consequence, the sonar is often at least 0.5 metres away from the patient. This 465 problem also happens with similar radio frequency technology used to monitor the body 466 movements and breathing.

467 One of the main factors that influence the accuracy of sleep detection devices is the quality of 468 their sensors. An informative discussion and comparison of sensors' accuracy appears in [10]. 469 Another important factor is the software that process the data collected by the sensor. Currently, 470 there are more than 100,000 health apps in the Apple and Google Play app stores [44]. Many of 471 these apps focus on sleep, and a large proportion of them implement proprietary sleep detection 472 algorithms. As a consequence, the same device (e.g., a mobile phone with an accelerometer) can 473 produce different results depending on the underlying software that process the data collected.

It is therefore very important to highlight that most of the publicly available sleep apps have not been clinically validated. Most of them are implemented and maintained by independent (non-clinical) programmers and, thus, their clinical use is not recommended. Of course, there have been many studies devoted to validating hardware devices and reporting on their accuracy and precision. Some studies devoted to validating actigraphs are $[45,46,47,48,49,50,51,52,53$, 54]. They all study the correlation between one commercial actigraph and a PSG (the patient wore the actigraph during the PSG). The sensitivity reported is in the interval $86 \%-98 \%$, and the specificity is in the interval $20 \%-54 \%$, because commercial actigraphs prioritize sensitivity over specificity. The interested reader is referred to [42] and [12] where systematic reviews of validation studies for sleep detection hardware devices can be found. Other works study the precision of actigraphy with specific populations (children, adults, old women, mentally disordered, etc.) $[55,56,49,57,58,59,54]$.

The review of the state of the technology together with the review of the validation studies advise against using contactless devices in the clinical study of sleep. Their low precision renders them far from being a reliable method. This does not mean that they are useless. They are good sleep indicators, and a good resource for patients to monitor and be aware of their own sleep quality. But their use as a definitive diagnostic tool is to be discouraged. In the case of contact devices, their precision is acceptable for many populations. In general, they should be used as an indicator but not as a definitive diagnostic tool, because several studies report that their sensitivity can fall down to $86 \%$ and their specificity to $20 \%$. However, these numbers are on the increase because the advances in technology are continuously improving such devices. This is also observable in the continuous increment of precision reported over time by validation studies.

\section{Conclusions}

The first conclusion of this review is that a perfect sleep assessment method does not exist. All methods have advantages and disadvantages, thus, they should be combined and adapted to the specific applicable needs. In terms of accuracy, the PSG is the best method, reporting the most complete and precise information (e.g., differentiating the sleep phases). Nevertheless, PSGs are expensive, exclusive (they require special hardware and medical support), can only be administered once, or for a few days, and they assess sleep in a stressful context (e.g., a hospital with video cameras recording and several machines registering the information provided by electrodes placed in the patient's body).

For these reasons, sleep diaries and questionnaires are often used to complement the PSG. They provide information that is gathered over medium to long periods of time, including information about sleep habits. Because they are mostly subjective, they have been erroneously considered as unreliable. But, in contrast, several studies (see Table 4) have proven that their 
509 sensitivity is often above $90 \%$, and between $73 \%$ and $97.7 \%$ in all the discussed studies.

510 Specificity ranges in the interval 50\%-96\%. In the specific case of electronic diaries, the studies

511 demonstrate that they produce the same results as their paper counterparts, but also that they

512 provide functional advantages: automatic data processing, metadata such as information about

513 when the patient filled in the diary, alerts, etc. Sleep questionnaires and diaries have been

514 classified in Table 2 and Table 5, respectively.

515 The literature review shows that the accuracy of hardware devices is superior to that of 516 questionnaires. This superiority, however, must be considered only in terms of precision, but not 517 in terms of diagnostic usefulness. The information provided by questionnaires regarding self518 perception of sleep quality is essential and cannot be replaced by hardware measures. The 519 sensitivity of hardware devices is $88-98 \%$, while their specificity is $20-52 \%$. The adherence of 520 hardware devices is also superior, because they require less effort from the patient (e.g.,

521

522

523

524

525

526

527

528

529

530

531

532

533

534

535

536

537

538

539

540

541

542

543 actigraphs are mostly automatic). The accuracy and reliability of hardware devices have been continuously increased with the advances of the technology. The continuous improvement of sensors and the appearance of new technologies (good examples are the imminent use of infrared thermometers and lidars) clearly improve the sleep detection devices.

We have presented a taxonomy of sleep methods that comprises all methods presented. This taxonomy classifies hardware devices into contact and contactless devices, because their functionality, accuracy, and reliability are different. In both cases, many studies reach the similar conclusion that current sleep trackers are useful tools to assess sleep and have been used successfully in many sleep studies. In particular, the studies that have evaluated concrete actigraphs with their respective software/app conclude that actigraphy is a reasonably reliable method to detect sleep with an average sensibility higher than $90 \%$. Nevertheless, the studies also report that the results obtained are particularly influenced by the patient that wears the actigraphy, which can produce bad results in many cases. Therefore, the use of actigraphy as a diagnostic tool should be complemented and contrasted with other methods to produce a more definitive diagnostic.

Finally, sleep apps are another important tool to assess sleep, especially in smartphones, where they are becoming very common nowadays. We have shown the most significant apps with regard to their number of reviews, and to their overall mark given by users. It is important to highlight that, contrasted with hardware, software apps are often implemented by independent (non-clinical) developers, and they do not pass any quality test. Therefore, they must be validated, at least before they are applied in clinically. The few scientific validation studies that have compared smartphone apps against PSG report that they are still not accurate enough to be used as clinical tools.

\section{Abbreviations List}

ARI: arousal index; ARP: arouse period; ASQ: Athens sleep questionnaire; AWI: awakening index; AWP: awake period; BQ: Berlin questionnaire; BIS: Bergen insomnia scale; BIPAP: bi level positive airway pressure; CPAP: continuous positive airway pressure; CSD: consensus sleep diary; CTT: CPAP titration test; EEG: electroencephalogram; EKG: electrocardiogram; EMG: electromyogram; EOG: electrooculogram; ESS: Epworth sleepiness scale; FS: final sleep; FOB: final out bed time; FOSQ: functional outcomes of sleep questionnaire; GASP: graduated apnea screening protocol; GERD: gastroesophageal reflux disease; GSH: get self help sleep diary; HST: home sleep test; IBT: in bed time; IIB: initial in bed time; ISI: insomnia severity 
553 index; ISL: initial sleep latency; LIDAR: light detection and ranging; LN: lights on time; LSRC: 554 Loughborough sleep research center; LT: lights out time; MAL: mean awakening length; MSL: 555 mean sleep latency; MSLT: multiple sleep latency test; MWT: maintenance of wakefulness test; 556 NHLBI: national heart, lung, and blood institute; NPS: MedicineWise sleep diary; NSF: national 557 sleep foundation; OSA50: obesity, snoring, apneas, aged over 50; OSAHS: sleep apnea 558 hypopnea syndrome; OSQ: Oviedo sleep questionnaire; PLMS: periodic limb movements during 559 sleep; PSD: Pittsburgh sleep diary; PSG: polysomnogram; PSQI: Pittsburgh sleep quality index; 560 QoL: quality of life index; RADAR: radio detection and ranging; ROC: receiver operating 561 characteristic; SACS: sleep apnea clinical score; SAQLI: Calgary sleep apnea quality of life 562 index; SBQ: STOP-BANG questionnaire; SDB: sleep-disordered breathing; SDQ: sleep 563 disorders questionnaire; SE: sleep efficiency; SEMSA: self-efficacy in sleep apnea; SFV: simple 564 four variables; SI: sleep interval; SL: sleep latency; SO: sleep onset; SONAR: sound navigation 565 and ranging; SP: sleep period; SQ: STOP questionnaire; TOB: time out of bed; TRT: total 566 recording time; TST: total sleep time; TWT: total wake time; WASO: wake after sleep onset. version of this article. His help to improve our work is greatly appreciated.

\section{References}

[1] Lomeli HA, Pérez-Olmos I, Talero-Gutiérrez C, Moreno CB, González-Reyes R, Palacios L, de la Peña F, Muñoz-Delgado J. 2008. Sleep evaluation scales and questionnaires: a review. Actas Españolas de Psiquiatría 36(1):50-9.

[2] Jessica MK, Strecker RE, Bianchi MT. 2012. Recent developments in home sleep-monitoring devices. ISRN Neurology 2012:768794.

[3] Winter C. 2014. Personal Sleep Monitors: Do They Work? The huffington post 2014 April 28.

[4] Silva GE, Vana KD, Goodwin JL, Sherrill DL, Quan SF. 2011. Identification of patients with sleep disordered breathing: comparing the four-variable screening tool, STOP, STOP-Bang, and Epworth Sleepiness Scales. Journal of Clinical Sleep Medicine 7(5):467-72.

[5] Firat H, Yuceege M, Demir A, Ardic S. 2012. Comparison of four established questionnaires to identify highway bus drivers at risk for obstructive sleep apnea in Turkey. Sleep and Biological Rhythms 10(3): 231-236.

[6] El-Sayed IH. 2012. Comparison of four sleep questionnaires for screening obstructive sleep apnea. Egyptian Journal of Chest Diseases and Tuberculosis 61(4): 433-441.

[7] Pataka A, Daskalopoulou E, Kalamaras G, Fekete Passa K, Argyropoulou P. 2014. Evaluation of five different questionnaires for assessing sleep apnea syndrome in a sleep clinic. Sleep Medicine 15(7):776-81.

[8] Singh J, Mims N. 2015. Screening Tools for the Obstructive Sleep Apnea for the Cardiovascular Clinician. American College of Cardiology, 2015 Jul 14.

[9] Adams R, Appleton S, Vakulin A, Martin SA, Grant JF, Antic NA, Catcheside P, Taylor AW, Wittert GA, McEvoy RD. 2015. Comparison of the OSA50, STOP and STOP-BANG Questionnaires for Identification of Obstructive Sleep Apnea in a Male Population Cohort. American Journal of Respiratory and Critical Care Medicine 191:A5601.

[10] Lee J, Finkelstein J. 2015. Consumer sleep tracking devices: a critical review. Studies in Health Technology and Informatics 210:45860.

[11] Ong AA, Gillespie MB. 2016. Overview of smartphone applications for sleep analysis. World Journal of Otorhinolaryngology-Head and Neck Surgery 2(1):45-49.

[12] Kolla BP, Mansukhani S, Mansukhani MP. 2016. Consumer sleep tracking devices: a review of mechanisms, validity and utility. Expert Review of Medical Devices 13(5):497-506.

[13] Maslakovic M. 2017. Ten gadgets for advanced sleep monitoring. Available at http://gadgetsandwearables.com/2017/01/09/the-bestsleep-trackers/ (accessed 9 January 2017).

[14] Green E. 2017. The 10 Best Sleep Trackers in 2017. Available at http://www.nosleeplessnights.com/best-sleep-tracker/ (accessed 6 January 2017).

[15] Robertson B, Marshall B, Carno MA. 2014. Polysomnography for the Sleep Technologist. Elsevier, ISBN: 9780323100199.

[16] Pandi-Perumal SR, Spence DW, BaHammam AS. 2014. Polysomnography: An Overview. Primary Care Sleep Medicine, 2014 July, $29-42$.

[17] Armon C, Johnson KG, Roy A, Nowack WJ. 2016. Polysomnography. Medscape, 2016 February 23, http://www.medscape.com/.

[18] Luo J, Huang R, Zhong X, Xiao Y, Zhou J. 2014. STOP-Bang questionnaire is superior to Epworth sleepiness scales, Berlin questionnaire, and STOP questionnaire in screening obstructive sleep apnea hypopnea syndrome patients. Chinese Medical Journal 127(17):3065-70.

[19] Silva GE, Goodwin JL, Vana KD, Quan SF. 2016. Obstructive sleep apnea and quality of life: comparison of the SAQLI, FOSQ, and SF-36 questionnaires. Southwest Journal on Pulmonary Critical Care 13(3):137-49.

[20] Sateia MJ. 2014. International Classification of Sleep Disorders-Third Edition. Chest Journal 146(5):1387-1394. 
[21] Carskadon MA. 1986. Guidelines for the Multiple Sleep Latency Test (MSLT): A Standard Measure of Sleepiness. Sleep 9(4):519524.

[22] Sullivan SS, Kushida CA. 2008. Multiple Sleep Latency Test and Maintenance of Wakefulness Test. Chest Journal 134(4):854-861.

[23] Banks S, Barnes M, Tarquinio N, Pierce RJ, Lack LC, McEvoy RD. 2004. The maintenance of wakefulness test in normal healthy subjects. Sleep 27(4):799-802.

610

611

612

613

614

615

616

617

618

[24] Meira L, van Zeller M, Eusébio E, Santa Clara E, Viana P, Drummond M. 2017. Maintenance of Wakefulness Test in clinical practice. ERJ Open Research 27(3):P5.

[25] Lopez-Campos JL, Garcia Polo C, Leon Jimenez A, Gonzalez-Moya E, Arnedillo A, Fernandez Berni JJ. 2007. CPAP titration: Different methods for similar clinical results. European Journal of Internal Medicine 18(3):230-234.

[26] Cruz SD, Littner MR, Zeidler MR. 2014. Home sleep testing for the diagnosis of obstructive sleep apnea-indications and limitations. Semin Respir Crit Care Med 35(5):552-9.

[27] Kapoor M, Greenough G. 2015. Home Sleep Tests for Obstructive Sleep Apnea (OSA). J Am Board Fam Med 28(4):504-9.

[28] Ibáñez V, Silva J, Cauli O. 2018. A survey on sleep questionnaires and diaries. Sleep medicine 42:90-96.

[29] Monk TH, Reynolds CF, Kupfer DJ, Buysse DJ, Coble PA, Hayes AJ, MacHen MA, Petrie SR, Ritenour AM. 1994. The Pittsburgh Sleep Diary. Journal of Sleep Research 3(2):111-120.

[30] Weitzman ED, Czeisler CA, Zimmerman JC, Ronda JM, Knauer RS. 1982. Chronobiological disorders: Analytic and therapeutic techniques. In: C. Guilleminault (Ed.) Sleeping and Waking Disorders: Indications and Techniques. Addison-Wesley, Menlo Park, CA.

[31] Carney CE, Buysse DJ, Ancoli-Israel S, Edinger JD, Krystal AD, Lichstein KL, Morin CM. 2012. The Consensus Sleep Diary: Standardizing Prospective Sleep Self-Monitoring. Sleep 35(2):287-302.

[32] Tonetti L, Mingozzi R, Natale V. 2016. Comparison between paper and electronic sleep diary. Biological Rhythm Research 47(5):743-753.

[33] Nakano H, Hirayama K, Sadamitsu Y, Toshimitsu A, Fujita H, Shin S, Tanigawa T. 2014. Monitoring sound to quantify snoring and sleep apnea severity using a smartphone: proof of concept. Journal of Clinical Sleep Medicine 10(1):73-8.

[34] Lee J, Hong M, Ryu S. 2015. Sleep Monitoring System Using Kinect Sensor. International Journal of Distributed Sensor Networks. October 25, 2015.

[35] Langley H. 2017. Counting sheep: The best sleep trackers and monitors. Available at https://www.wareable.com/withings/best-sleeptrackers-and-monitors (accessed 7 February 2017).

[36] ASA. 2017. Sleep Devices. American Sleep Association (ASA). URL: https://www.sleepassociation.org/sleep-devices/.

[37] Hacktosleep. 2016. The 6 Best Apps For Tracking Your Sleep. Available at https://hacktosleep.com/the-6-best-apps-for-trackingyour-sleep/ (accessed 22 August 2016).

[38] Patel P, Kim JY, Brooks LJ. 2017. Accuracy of a smartphone application in estimating sleep in children. Sleep Breath 21(2):505-511.

[39] Lorenz CP, Williams AJ. 2017. Sleep apps: what role do they play in clinical medicine? Curr Opin Pulm Med 23(6):512-516.

[40] Tryon W, Bellak A, Hersen M. 1991. Activity Measurement in Psychology and Medicine. New York, NY: Plenum Press, 1991.

[41] Boyne K, Sherry DD, Gallagher PR, Olsen M, Brooks LJ. 2013. Accuracy of computer algorithms and the human eye in scoring actigraphy. Sleep Breath 17(1):411-7. DOI: 10.1007/s11325-012-0709-z.

[42] Evenson KR, Goto MM, Furberg RD. 2015. Systematic review of the validity and reliability of consumer-wearable activity trackers. International Journal of Behavioral Nutrition and Physical Activity, 2015 December, 12:159.

[43] Jungquist CR, Pender JJ, Klingman KJ, Mund J. 2015. Validation of Capturing Sleep Diary Data via a Wrist-Worn Device. Sleep Disorders, 2015, Article ID 758937.

[44] Research 2 Guidance. 2016. mHealth App Developer Economics 2016. Available at www.research2guidance.com (accessed 16 October 2016).

[45] Sivertsen B, Omvik S, Havik OE, Pallesen S, Bjorvatn B, Nielsen GH, Straume S, Nordhus IH. 2006. A comparison of actigraphy and polysomnography in older adults treated for chronic primary insomnia. Sleep 29(10):1353-1358.

[46] Paquet J, Kawinska A, Carrier J. 2007. Wake detection capacity of actigraphy during sleep. Sleep 30(10):1362-1369.

[47] Sitnick SL, Goodlin-Jones BL, Anders TF. 2008. The use of actigraphy to study sleep disorders in preschoolers: some concerns about detection of nighttime awakenings. Sleep 31(3):395-401.

[48] Montgomery-Downs HE, Insana SP, Bond JA. 2012. Movement toward a novel activity monitoring device. Sleep and Breathing 16(3):913-7.

[49] Marino M, Li Y, Rueschman MN, Winkelman JW, Ellenbogen JM, Solet JM, Dulin H, Berkman LF, Buxton OM. 2013. Measuring sleep: accuracy, sensitivity, and specificity of wrist actigraphy compared to polysomnography. Sleep 36(11):1747-55.

[50] Meltzer LJ, Hiruma LS, Avis K, Valentin J. 2014. Comparison of a Commercial Accelerometer with Polysomnography and Actigraphy in Children and Adolescents. Sleep 38(8).

[51] De Zambotti M, Baker FC, Colrain IM. 2015a. Validation of Sleep-Tracking Technology Compared with Polysomnography in Adolescents. Sleep 38(9):1461-8.

[52] Bhat S, Ferraris A, Gupta D, Mozafarian M, DeBari VA, Gushway-Henry N, Gowda SP, Polos PG, Rubinstein M, Seidu H, Chokroverty S. 2015. Is there a clinical role for smartphone seep apps? Comparison of sleep cycle detection by a smartphone application to polysomnography. Journal of Clinical Sleep Medicine 11:709-715.

[53] Toon E, Davey MJ, Hollis SL, Nixon GM, Horne RSC, Biggs SN. 2016. Comparison of Commercial Wrist-Based and Smartphone Accelerometers, Actigraphy, and PSG in a Clinical Cohort of Children and Adolescents. Journal of Clinical Sleep Medicine 12(3): 343-350.

[54] Meltzer LJ, Wong P, Biggs SN, Traylor J, Kim JY, Bhattacharjee R, Narang I, Marcus CL. 2016. Validation of Actigraphy in Middle Childhood. Sleep 39(6):1219-24.

[55] Blackwell T, Redline S, Ancoli-Israel S, Schneider JL, Surovec S, Johnson NL, Cauley JA, Stone KL. 2008. Comparison of sleep parameters from actigraphy and polysomnography in older women: the SOF study. Sleep 31(2):283-91.

[56] Martin JL, Hakim AD. 2011. Wrist Actigraphy. Chest 139(6):1514-1527.

[57] Baandrup L, Jennum PJ. 2015. A validation of wrist actigraphy against polysomnography in patients with schizophrenia or bipolar disorder. Journal of Neuropsychiatric Disease and Treatment 11:2271-2277.

[58] Min JK, Doryab A, Wiese J, Amini S, Zimmerman J, Hong JI. 2014. Toss 'n' turn: smartphone as sleep and sleep quality detector. Proceedings of the SIGCHI Conference on Human Factors in Computing Systems. ACM 2014. pp. 477-486. 
677

678

679

680

681

682

683

684

685

686

687

688

689

690

691

692

693

694

695

696

697

698

699

700

701

702

703

704
[59] De Zambotti M, Claudatos S, Inkelis S, Colrain IM, Baker FC. 2015b. Evaluation of a consumer fitness-tracking device to assess sleep in adults. Chronobiology International 32(7):1024-8.

[60] Zoomer J, Peder R, Rubin AH, Lavie P. 1985. Mini Sleep Questionnaire for screening large populations for EDS complaints. In: Koella WP, Ruther E, Schulz H (eds). Sleep '84. Gustav Fisher, Stuttgart, 1985; 467-470.

[61] Buysse DJ, Reynolds CF, Monk TH, Berman SR, Kupfer DJ. 1989. The Pittsburgh Sleep Quality Index: a new instrument for psychiatric practice and research. Psychiatry Res. 28(2):193-213.

[62] Johns MW. 1991. A new method for measuring daytime sleepiness: the Epworth sleepiness scale. Sleep 14(6):540-5.

[63] Morin CM. 1993. Insomnia: psychological assessment and management. New York: Guilford Press, 1993.

[64] Douglass AB, Bornstein R, Nino-Murcia G, Keenan S, Miles L, Zarcone VP Jr, Guilleminault C, Dement WC. 1994. The Sleep Disorders Questionnaire. I: Creation and multivariate structure of SDQ. Sleep 17(2):160-7.

[65] Flemons WW, Whitelaw WA, Brant R, Remmers JE. 1994. Likelihood ratios for a sleep apnea clinical prediction rule. American Journal of Respiratory and Critical Care Medicine 150(5):1279-1285.

[66] Weaver TE, Laizner AM, Evans LK, Maislin G, Chugh DK, Lyon K, Smith PL, Schwartz AR, Redline S, Pack AI, Dinges DF. 1997. An instrument to measure functional status outcomes for disorders of excessive sleepiness. Sleep 20(10):835-43.

[67] Flemons WW, Reimer MA. 1998. Development of a disease-specific health-related quality of life questionnaire for sleep apnea. American Journal of Respiratory and Critical Care Medicine 158(2):494-503.

[68] Bobes J, González MP, Vallejo J, Sáiz J, Gibert J, Ayuso JL, Rico F. 1998. Oviedo Sleep Questionnaire (OSQ): A new semistructured Interview for sleep disorders. European Neuropsychopharmacology 8(2):S162.

[69] Netzer NC, Stoohs RA, Netzer CM, Clark K, Strohl KP. 1999. Using the Berlin Questionnaire to identify patients at risk for the sleep apnea syndrome. Annals of internal medicine 131(7):485-91.

[70] Soldatos CR, Dikeos DG, Paparrigopoulos TJ. 2000. Athens Insomnia Scale: validation of an instrument based on ICD-10 criteria. Journal of Psychosomatic Research 48:555-560.

[71] Weaver TE, Maislin G, Dinges DF, Younger J, Cantor C, McCloskey S, Pack AI. 2003. Self-efficacy in sleep apnea: instrument development and patient perceptions of obstructive sleep apnea risk, treatment benefit, and volition to use continuous positive airway pressure. Sleep 26(6):727-32.

[72] Chung F, Yegneswaran B, Liao P, Chung S, Vairavanathan S, Islam S, Khajehdehi A, Shapiro C. 2008. STOP questionnaire. A tool to screen patients for obstructive sleep apnea. Anesthesiology 108(5):812-21.

[73] Pallesen S, Bjorvatn B, Nordhus IH, Sivertsen B, Hjørnevik M, Morin CM. 2008. A new scale for measuring insomnia: the Bergen Insomnia Scale. Perceptual and Motor Skills 107(3):691-706.

[74] Chasens ER, Ratcliffe SJ, Weaver TE. 2009. Development of the FOSQ-10: a short version of the functional outcomes of sleep questionnaire. Sleep 32(7):915-919.

[75] Takegami M, Hayashino Y, Chin K, Sokejima S, Kadotani H, Akashiba T, Kimura H, Ohi M, Fukuhara S. 2009. Simple four-variable screening tool for identification of patients with sleep-disordered breathing. Sleep 32(7):939-48.

[76] Chai-Coetzer CL, Antic NA, Rowland LS, Catcheside PG, Esterman A, Reed RL, Williams H, Dunn S, McEvoy RD. 2011. A simplified model of screening questionnaire and home monitoring for obstructive sleep apnea in primary care. Thorax 66(3):213-9. 


\section{Table $\mathbf{1}$ (on next page)}

Definition of basic sleep assessment parameters

This table summarizes the main parameters of a sleep study. The top of the table (grey rows) lists the fundamental parameters. Those parameters that can be derived from the primitive variables are listed in the white rows. Each of them includes its associated formula. 
1 Table 1. Definition of basic sleep detection parameters

\begin{tabular}{|c|c|c|c|}
\hline & Sleep Measure & Definition & Formula \\
\hline \multirow{11}{*}{ 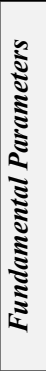 } & Initial In Bed Time (IIB) & Time when patient goes to bed initially & - \\
\hline & Final Out Bed Time (FOB) & Time when patient leaves the bed definitely & - \\
\hline & Time Out of Bed (TOB) & Total time out of bed between IIB and FOB & - \\
\hline & Lights Out Time (LT) & Time of lights out & - \\
\hline & Lights On Time $(L N)$ & Time of lights on & - \\
\hline & Sleep Onset ( $\mathrm{SO}$ ) & Time when first sleep starts & - \\
\hline & Final Sleep (FS) & Time when last sleep finishes & \\
\hline & Sleep Latency $(S L)$ & Time taken to fall sleep (at any time) & - \\
\hline & Sleep Period (SP) & Time spent sleeping between two awakenings/SO & - \\
\hline & Awake Period (AWP) & Time spent awake between two sleep periods & (awakening $=$ wake period $>10 \mathrm{~s}$ ) \\
\hline & Arouse Period (ARP) & Time spent awake between two sleep periods & (arousal $=$ wake period $<10 \mathrm{~s}$ ) \\
\hline \multirow{12}{*}{ 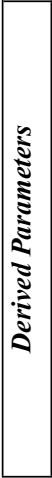 } & In Bed Time (IBT) & Total time in bed & $\mathrm{IBT}=\mathrm{FOB}-\mathrm{IIB}-\mathrm{TOB}$ \\
\hline & Total Recording Time (TRT) & Time between lights out and lights on & TRT $=$ LN-LT \\
\hline & Initial Sleep Latency (ISL) & Time taken to fall sleep the first time & $\mathrm{ISL}=\mathrm{SO}-\mathrm{LT}$ \\
\hline & Total Sleep Time (TST) & Amount of time the patient sleeps during TRT & $\begin{aligned} & \mathrm{TST}=\sum_{i=1}^{i=N(\# \text { sleep periods) }} S P_{i} \\
&\end{aligned}$ \\
\hline & Sleep Interval (SI) & Time between the first sleep and the last sleep & $\mathrm{SI}=\mathrm{FS}-\mathrm{SO}$ \\
\hline & Wake After Sleep Onset (WASO) & Wake time between IIB and FOB & WASO $=$ SI-TST \\
\hline & Total Wake Time (TWT) & All wake time throughout TRT & TWT $=$ ISL+WASO \\
\hline & Mean Sleep Latency (MSL) & Arithmetic average of sleep latencies & $\mathrm{MSL}=\left(\sum_{i=1}^{i=N(\# \text { sleep latencies })} S L_{i}\right) / N$ \\
\hline & Sleep Efficiency (SE) & Percentage of sleep of the total time in bed & $\mathrm{SE}=(\mathrm{TST} / \mathrm{TRT}) \times 100$ \\
\hline & Mean Awakening Length (MAL) & Arithmetic average of awake periods & MAL $=\left(\sum_{i=1}^{i=N(\# \text { awake periods })} A W P_{i}\right) / N$ \\
\hline & Awakening Index (AWI) & Number of awakenings per unit of time & $\mathrm{AWI}=\# \mathrm{AWP} / \mathrm{TST}$ \\
\hline & Arousal Index (ARI) & Number of arousals per unit of time & $\mathrm{ARI}=$ \#ARP/TST \\
\hline
\end{tabular}




\section{Table 2 (on next page)}

Questionnaires for the detection of sleep disorders

Each row represents a sleep questionnaire, and includes its acronym, its structure (number of items and scale used), and a reference to the article where it was proposed. 
1 Table 2. Questionnaires for the detection of sleep disorders

\begin{tabular}{|c|c|c|c|c|}
\hline \multicolumn{2}{|r|}{ Sleep Questionnaire } & Structure & Period & Objectivity \\
\hline$M S Q$ & Mini Sleep Questionnaire [60] & 10 items ( 7 points scale) & Recently & 0 \\
\hline PSQI & Pittsburgh Sleep Quality Index [61] & 9 items (4 points scale) & 1 month & 0 \\
\hline ESS & Epworth Sleepiness Scale [62] & 8 items (4 points scale) & Recently & 0 \\
\hline$I S I$ & Insomnia Severity Index [63] & 7 items ( 5 points scale) & Recently & 0 \\
\hline$S D Q$ & Sleep Disorders Questionnaire [64] & 175 items ( 5 points scale) & Recently & 1 \\
\hline$S A C S$ & Sleep apnea clinical score [65] & 4 items (100 points scale) & Recently & 4 \\
\hline$F O S Q$ & Functional Outcomes of Sleep Questionnaire [66] & 30 items (4-5 points scale) & Recently & 0 \\
\hline$S A Q L I$ & Calgary Sleep Apnea Quality of Life Index [67] & 35 items ( 7 points scale) & 1 month & 0 \\
\hline$O S Q$ & Oviedo Sleep Questionnaire [68] & 15 items (4-7 points scale) & 1 month & 0 \\
\hline$B Q$ & Berlin Questionnaire [69] & 10 items ( $2-5$ points scale) & Recently & 2 \\
\hline$A S Q$ & Athens Sleep Questionnaire [70] & 8 items (4 points scale) & 1 month & 0 \\
\hline SEMSA & Self-efficacy in Sleep Apnea [71] & 26 items (4 points scale) & Recently/Future & 0 \\
\hline SQ & STOP Questionnaire [72] & 4 items ( 2 points scale) & Recently & 2 \\
\hline$S B Q$ & STOP-BANG Questionnaire [73] & 8 items ( 2 points scale) & Recently & 3 \\
\hline$B I S$ & Bergen Insomnia Scale [74] & 6 items ( 8 points scale $)$ & 1 month & 0 \\
\hline$F O S Q-10$ & Functional Outcomes of Sleep Questionnaire - 10 [75] & 10 items (4 points scale) & Recently & 0 \\
\hline$S F V$ & Simple Four Variables [76] & 4 items (2-6 points scale) & Recently & 3 \\
\hline OSA50 & Obesity, Snoring, Apneas, aged over 50 [77] & 4 items (3-4 points scale) & Recently & 4 \\
\hline
\end{tabular}




\section{Table 3 (on next page)}

Purposes of sleep questionnaires

Each row represents a sleep questionnaire, and it indicates what does this questionnaire intend to measure. 
1 Table 3. Purposes of sleep questionnaires

\begin{tabular}{|l|l|l|}
\hline \multicolumn{1}{|c|}{ Sleep Questionnaire } & Acronym & \multicolumn{1}{c|}{ Measures } \\
\hline Mini Sleep Questionnaire & $M S Q$ & Insomnia and hypersomnia \\
\hline Pittsburgh Sleep Quality Index & PSQI & Sleep quality and patterns of sleep in adults \\
\hline Epworth Sleepiness Scale & ESS & Level of daytime sleepiness. Average sleep propensity in daily life \\
\hline Insomnia Severity Index & $I S I$ & Nature, severity, and impact of insomnia. Treatment response in adults \\
\hline Sleep Disorders Questionnaire & $S D Q$ & Sleep disturbance and usual sleep habits during the past month only \\
\hline Sleep apnea clinical score & SACS & Sleep apnea \\
\hline Functional Outcomes of Sleep Questionnaire & FOSQ & Impact of excessive sleepiness on daily life \\
\hline Calgary Sleep Apnea Quality of Life Index & SAQLI & Quality of life associated with sleep apnea \\
\hline Oviedo Sleep Questionnaire & OSQ & Insomnia and hypersomnia in the last month \\
\hline Berlin Questionnaire & BQ & Sleep apnea \\
\hline Athens Sleep Questionnaire & ASQ & Sleep quality \\
\hline Self-efficacy in Sleep Apnea & SEMSA & Sleep apnea \\
\hline STOP Questionnaire & SQ & Sleep apnea \\
\hline STOP-BANG Questionnaire & SBQ & Sleep apnea \\
\hline Bergen Insomnia Scale & BIS & Sleep quality \\
\hline Functional Outcomes of Sleep Questionnaire - 10 & FOSQ-10 & Impact of excessive sleepiness on daily life \\
\hline Simple Four Variables & SFV & Sleep apnea \\
\hline Obesity, Snoring, Apneas, aged over 50 & OSA50 & Sleep apnea \\
\hline
\end{tabular}




\section{Table 4 (on next page)}

Studies that compare sleep assessment questionnaires

Each row represents a study that compares 3-5 sleep questionnaires. For each study, the table shows the size of the sample used (amount of people that participated in the study) and which questionnaire produced the best sensitivity and specificity. The reference to each study is also included. 
1 Table 4. Studies that compare sleep assessment questionnaires

\begin{tabular}{|c|c|c|c|c|}
\hline Questionnaires evaluated & Sample & Best sensitivity & Best specificity & Reference \\
\hline ESS vs. SQ vs. SBQ vs. SFV & 4770 & SBQ $(87.0 \%)$ & SFV $(93.2 \%)$ & Silva G.E. et al., 2011 [4] \\
\hline$E S S$ vs. $B Q$ vs. $S Q$ vs. $S B Q$ & 234 & SBQ $(97.55 \%)$ & $\operatorname{ESS}(75.0 \%)$ & El-Sayed I.H., 2012 [6] \\
\hline$B Q$ vs. $S Q$ vs. $S B Q$ vs. OSA50 & 90 & SBQ $(87 \%)$ & SBQ $(76.0 \%)$ & Firat H. et al., 2012 [5] \\
\hline$E S S$ vs. $B Q$ vs. $S Q$ vs. $S B Q$ & 212 & SBQ $(94.9 \%)$ & SFV $(50.0 \%)$ & Luo J. et al., 2014 [18] \\
\hline$E S S$ vs. $B Q$ vs. $S Q$ vs. $S B Q$ vs. $S F V$ & 1853 & SBQ $(97.6 \%)$ & $\operatorname{SFV}(74.4 \%)$ & Pataka A. et al., 2014 [7] \\
\hline$S Q$ vs. $S B Q$ vs. OSA50 & 543 & OSA50+oximetry $(73.0 \%)$ & OSA50+oximetry $(96.0 \%)$ & Adams R. et al., 2015 [9] \\
\hline
\end{tabular}

2 


\section{Table 5 (on next page)}

Sleep diaries for the detection of sleep problems

Each row represents a sleep diary, and it indicates the number of questions included in the diary and the scale used to complete the answers. 
1 Table 5. Sleep diaries for the detection of sleep problems

\begin{tabular}{|l|c|c|}
\hline \multicolumn{1}{|c|}{ Sleep Diary } & Number of Questions & Scale \\
\hline Pittsburgh Sleep Diary (PSD) & 23 & 6 points scale \\
\hline Consensus Sleep Diary (CSD) & 20 & 5 points scale \\
\hline National Sleep Foundation (NSF) & 15 & 3 points scale \\
\hline Get Self Help Sleep Diary (GSH) & 14 & 11 points scale \\
\hline National Heart, Lung, and Blood Institute (NHLBI) & 12 & $3-4$ points scale \\
\hline NPS MedicineWise Sleep Diary (NPS) & 11 & 3 points scale \\
\hline Loughborough Sleep Research Center (LSRC) & 8 & 5 points scale \\
\hline
\end{tabular}

2 


\section{Table 6(on next page)}

Contactless sleep detection apps (prices and reviews are taken from Google Play)

Each row shows a sleep app. Rows are sorted in descending order according to the average review mark. 
1 Table 6. Contactless sleep detection apps (prices and reviews are taken from Google Play)

\begin{tabular}{|l|c|c|c|c|}
\hline \multicolumn{1}{|c|}{ App name } & Developer & Price & Average review & Number of reviews \\
\hline Sleep as Android Unlock & Urbandroid Team & $3.99 \$$ & 4.5 out of 5 & 23,686 \\
\hline Sleep Cycle Alarm Clock & Northcube AB & $0 \$$ & 4.4 out of 5 & 47,965 \\
\hline Sleep as Android & Urbandroid Team & $0 \$$ & 4.3 out of 5 & 244,840 \\
\hline Sleep Better & Runtastic & $0 \$$ & 4.1 out of 5 & 108,825 \\
\hline Sleep Time & Azumio Inc. & $0 \$$ & 4.1 out of 5 & 30,418 \\
\hline Smart Sleep Manager & 株式会社 C2 & $0 \$$ & 4.1 out of 5 & 18,805 \\
\hline Good Night's Sleep Alarm & Ateam Inc. & $0 \$$ & 4.1 out of 5 & 10,022 \\
\hline SleepBot & SleepBot & $0 \$$ & 4.0 out of 5 & 51,111 \\
\hline Smart Alarm Clock & Nelurra Holdings LTD & $0 \$$ & 3.9 out of 5 & 27,442 \\
\hline Sleep Analyzer & A1 Brains Infotech & $0 \$$ & 3.1 out of 5 & 2,461 \\
\hline
\end{tabular}




\section{Figure 1}

QUOROM flow chart of the reviewing process

Solid arrows represent the QUOROM flow. Dashed arrows represent the decomposition of a box into several sub-boxes.

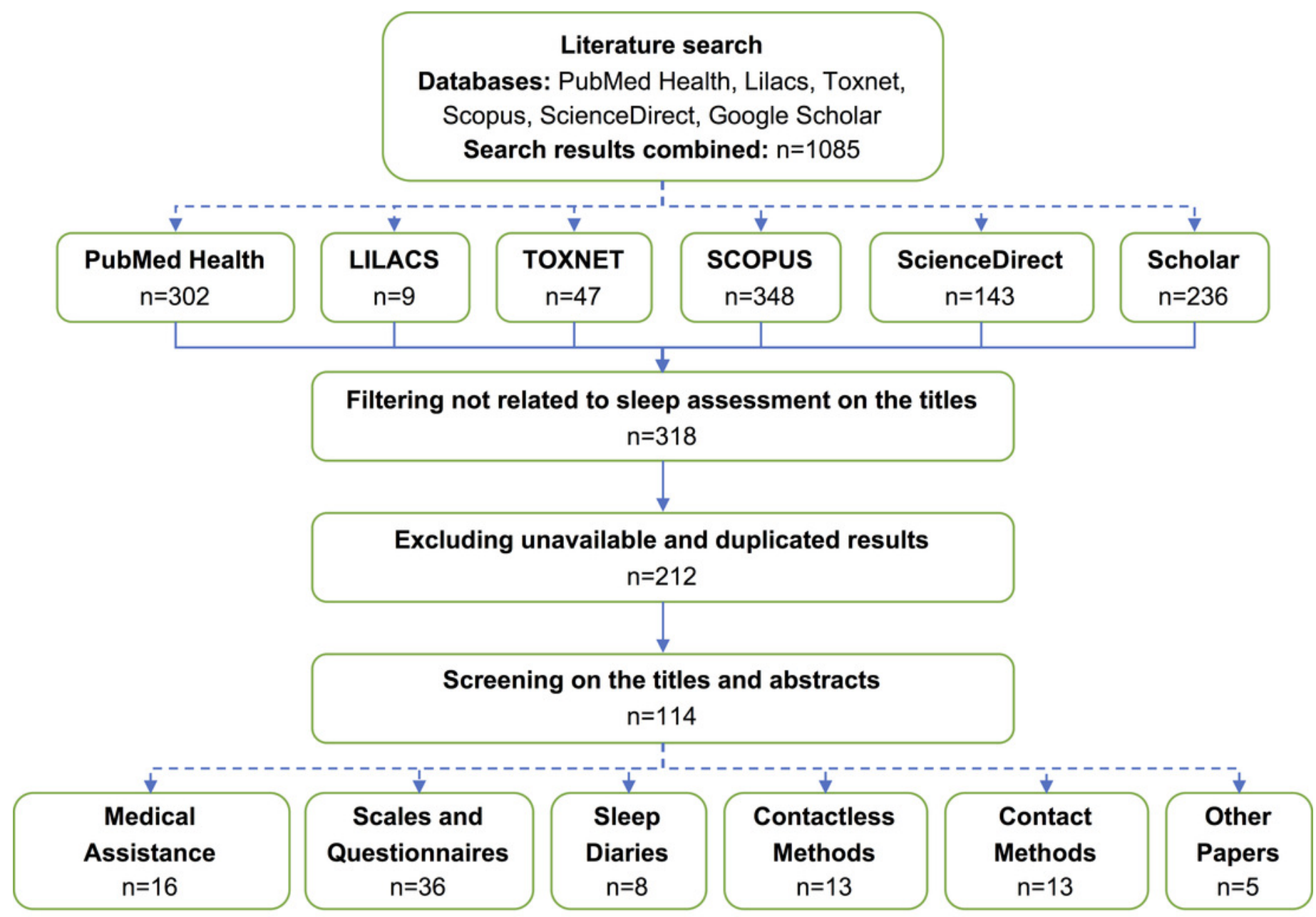


Figure 2

\section{Taxonomy of sleep detection methods}

Grey boxes represent categories. White boxes represent sleep assessment methods or technology used to assess sleep.

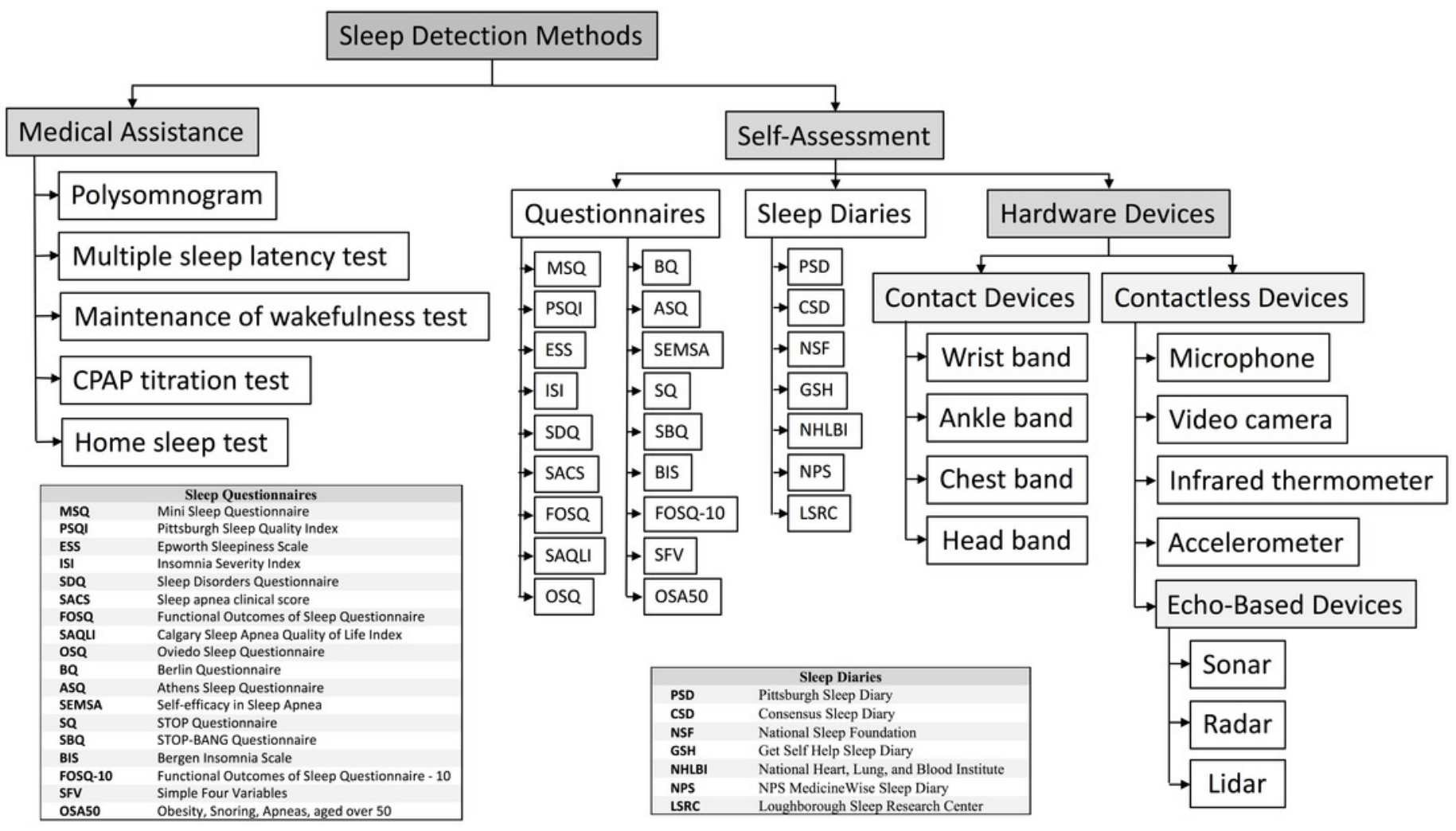




\section{Figure 3}

\section{Classification of the main disorders evaluated with a polysomnography}

The main disorders evaluated with a polysomnogram are structured with a three-levels taxonomy that follows the International Classification of Sleep Disorders.

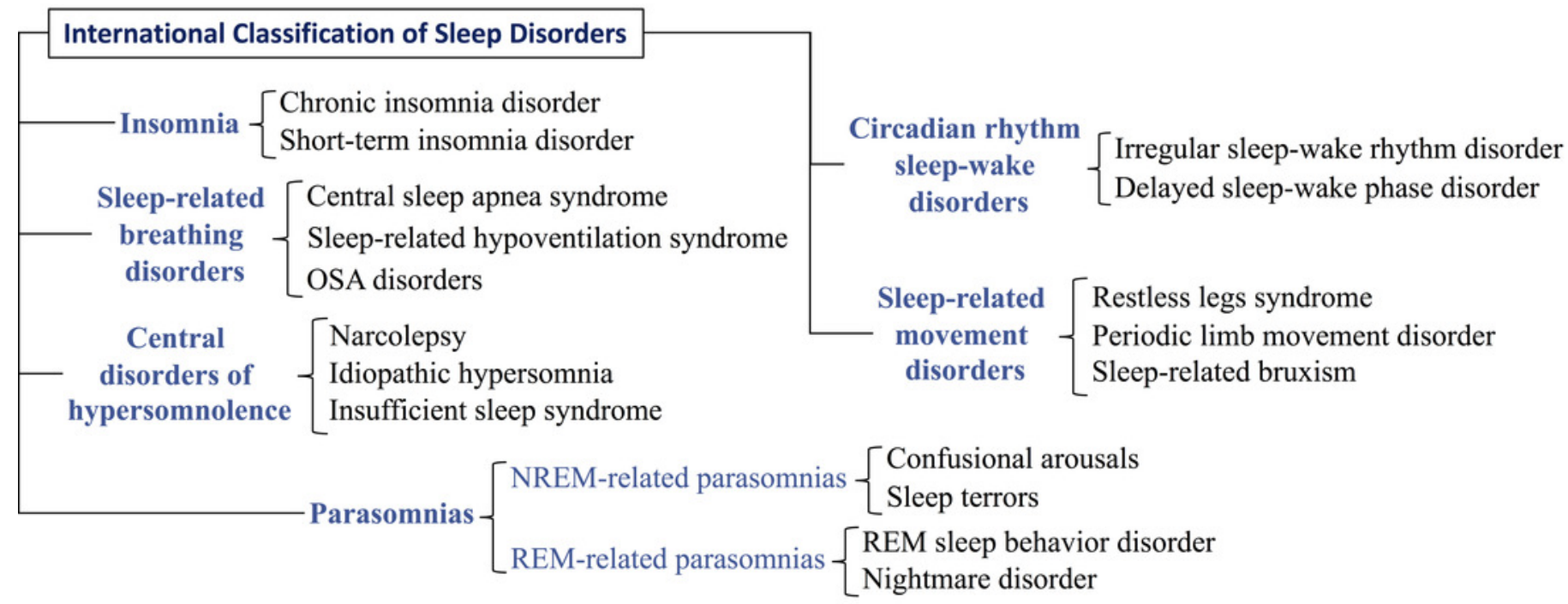

